\title{
Selective maintenance considering two types of failure modes
}

\author{
Mayank Pandey and Ming J. Zuo* \\ Department of Mechanical Engineering, \\ University of Alberta, \\ Edmonton, Alberta, T6G 2G8, Canada \\ E-mail: mayank@ualberta.ca \\ E-mail: ming.zuo@ualberta.ca \\ *Corresponding author
}

\begin{abstract}
Owing to limited maintenance resources, it may not be possible to perform all feasible maintenance actions on a system during the maintenance break between two successive missions. A subset of maintenance actions can then be selected from the available maintenance options so that the system can meet the next mission requirement. This maintenance policy is called selective maintenance. In this paper, selective maintenance has been performed for a system that has been subjected to the two types of failure modes: maintainable and the non-maintainable failure modes. The hazard rate due to the maintainable failure modes depends on the cumulative effect of the non-maintainable failure modes. A model has been used to show this relationship. An illustrative example has been used to demonstrate the effect of the two failure modes and the advantages of imperfect maintenance in selective maintenance decision making.
\end{abstract}

Keywords: selective maintenance; asset management; maintainable failure mode; non-maintainable failure mode; imperfect maintenance; differential evolution.

Reference to this paper should be made as follows: Pandey, M. and Zuo, M.J. (2014) 'Selective maintenance considering two types of failure modes', Int. J. Strategic Engineering Asset Management, Vol. 2, No. 1, pp.37-62.

Biographical notes: Mayank Pandey is a $\mathrm{PhD}$ candidate in the Department of Mechanical Engineering at the University of Alberta, Edmonton, Canada. $\mathrm{He}$ received his BTech in Mechanical Engineering from M.J.P. Rohilkhand University, Bareilly, India in 2005 and MTech in Manufacturing Engineering from NIFFT, Ranchi, India in 2007. His research interests include reliability modelling and application, maintenance modelling and scheduling, condition-based maintenance, selective maintenance and optimisation. He is a member of IEEE.

Ming J. Zuo received his BSc in Agricultural Engineering in 1982 from Shandong Institute of Technology, China, MSc in 1986 and PhD in 1989 both in Industrial Engineering from Iowa State University, Ames, Iowa, USA. He is currently a Full Professor in the Department of Mechanical Engineering at the University of Alberta, Canada. His research interests include system reliability analysis, maintenance modelling and optimisation, signal processing, and fault diagnosis. He is a Regional Editor for North and South American Region for International Journal of Strategic Engineering Asset Management (ISEAM). 
This paper is a revised and expanded version of a paper entitled 'Selective maintenance for a multi-component system with two types of failure modes under age-based imperfect maintenance', presented at the 19th ISSAT International Conference: Reliability and Quality in Design, Honolulu, Hawaii, USA, 5-7 August 2013.

\section{Introduction}

All equipment and systems deteriorate over time and need maintenance to improve their reliability and availability. If a system is not able to perform its intended function(s), it is said to have failed. According to Hoyland and Rausand (1994), "All technical items are designed to fulfill one or more functions; a failure mode is thus defined as non-fulfilment of one of these functions". Thus, if the corresponding function is unavailable, this is described as "failure with respect to a given failure mode of the system" (Zequeira and Bérenguer 2006). Preventive maintenance (PM) of a machine may include lubrication, tightening screws, cleaning and shaft alignment. But these PM activities can only influence the failure modes that are affected by these working conditions. PM activities can reduce the hazard rate of such failure modes. These failure modes are called maintainable failure modes. However, the hazard rate of the non-maintainable failure modes cannot be changed by PM activities. These failure modes are related to the inherent design of the system, as in a crack in a shaft or gear. For example, it is possible to maintain or replace some components of a system in order to prevent the failure modes relevant to these components. However, failures associated with the fatigue or wear of the system over time are usually not maintainable. One type of failure mode may be affected by another type of failure mode in the system (Huynh et al., 2011). Yang et al. (2012) suggested that maintenance decisions and maintenance resource allocations may be affected by the type and frequency of these failure modes. It is important to consider the interaction between different failure modes and determine their effect on system design, performance (Liu, 2012; Kim et al., 2013) and maintenance decision making (Liu et al., 2012).

Several examples have been reported in the literature in which different failures within a system are coupled. Zequeira and Bérenguer (2006) provided an example of the electric truck motor which is a complex series system. It consists of several components like armature, planetary gear and sun pinion. The failure of one component may affect the failure of another component in the system. For example, damage in the armature winding (a non-maintainable failure) may increase the system's temperature, which may cause in turn the lubrication to burn more quickly, resulting in inadequate lubrication (a maintainable failure). Zequeira and Bérenguer (2006) mentioned that the interdependence of failures can be used to study their effects on the overall system reliability. Another example would be the belt conveyor system, in which an idler bearing failure, which is a non-maintainable failure, may result in the slipping of the conveyor belt which is a maintainable failure mode. Similarly, the coating of the pulley wears off over a period of time, which is a non-maintainable type of damage. It may lead to increased friction and cause the belt joints to fail, which is a maintainable type of failure. Another example would be pulley bearing damage (a non-maintainable failure) that may lead to increased vibration that causes belt damage (a maintainable failure). 
Lin et al. (2001) used the concept of maintainable and non-maintainable failure modes. They scheduled maintenance activities for a system with these two types of failure modes. They assumed that the maintainable and non-maintainable failure modes were independent and that there was no interaction between the components in the system that were experiencing the non-maintainable and/or maintainable failures. However, interaction between the components in a system may exist that characterises system degradation behaviour. Zequeira and Bérenguer (2006) also mentioned that the interaction between the components can be used to characterise the system degradation. This interaction may include vibration or high temperature. They studied the maintainable and non-maintainable failure modes and suggested that there may be a relationship between the two failure modes that can be stated in terms of the hazard rates. They suggested that the hazard rate due to the maintainable failure modes is related to the hazard rate due to the non-maintainable failure modes. Such a relation allows one to take into account the possible interactions between the failure modes when the failure patterns are coupled. They proposed a relation between the hazard rates of the maintainable and non-maintainable failure modes by using a coupling function. However, determination of this function was difficult.

Castro (2009) proposed another relation between the hazard rates due to the maintainable and non-maintainable failure modes. He determined that the hazard rate due to the maintainable failure modes depends on the cumulative effect of the hazard rate due to the non-maintainable failure modes. Lin et al. (2001) determined that the hazard rate due to non-maintainable failure modes depends on the effective age of the component. However, Castro (2009) suggested that the hazard rate due to non-maintainable failure modes depends on the calendar age (excluding downtime) of the component up to the last maintenance. Both have their shortcomings. The effective age of a component is affected by the maintenance action with the result that the hazard rate due to the non-maintainable failure modes will be lower for an effective age-based model (Lin et al., 2001) than for a calendar age-based model (Castro, 2009). The latter is more logical because non-maintainable failure modes are not affected by a maintenance action and therefore the associated hazard rate is a continuously increasing function. If this hazard rate depends on the effective age, which may change after maintenance, it will no longer be a continuously increasing function. However, in Castro's (2009) study, the hazard rate of the maintainable failure modes during a mission was assumed to be dependent on the hazard rate of the non-maintainable failure modes up to the last maintenance only. He did not consider the interaction between these two hazard rate functions during the current mission. Therefore, the hazard rate due to the maintainable failure modes during the current mission evaluated by Castro (2009) is inaccurate. Chen et al. (2012) applied Castro's (2009) model and assumed that the cumulative hazard rate due to non-maintainable failure modes during the period immediately preceding the current calendar time affected the hazard rate due to maintainable failure modes. This consideration was also not logical. It is more reasonable to assume that non-maintainable failure modes up to the current calendar time affect the hazard rate due to maintainable failure modes. In this paper, we have proposed a model in which the hazard rate due to the maintainable failure modes depends on the instantaneous hazard rate due to the non-maintainable failure modes.

Maintenance is critical to system performance and its reliability. Maintenance strategy aims to determine a trade-off between profits and the maintenance budget (Wang, 2002). PM actions may increase the lifetime of a piece of equipment and decrease 
its breakdown frequency. Traditionally, it is assumed that the maintenance of a system can improve its condition to as good as new (AGAN, also called replacement) or as bad as old (ABAO also called minimal repair). However, this assumption is not always realised in practice. For example, if only a few components are replaced, the whole system can be considered to be in between AGAN and ABAO conditions (Pham and Wang, 2003). Such a maintenance policy is called imperfect maintenance. The system's effective age or hazard rate function can be adjusted to model the effect of a maintenance action (Malik, 1979; Nguyen and Murthy, 1981). Nakagawa (1988) introduced improvement factors in the hazard rate and the effective age so as to consider the effect of the maintenance. Lin et al. (2000) proposed that a maintenance action can simultaneously affect the effective age as well as the hazard rate of a system. They introduced a hybrid imperfect PM model to represent the effect of the maintenance in which the effective age of a system is reduced by a factor and the hazard rate due to maintainable failure modes increases by a factor after maintenance. Castro (2009) assumed that after maintenance, the system is restored to AGAN condition with respect to the maintainable failure modes.

Based on Castro's (2009) model, we have presented a preliminary work on selective maintenance with the two types of failure modes in Pandey et al. (2013a), where a system can be restored in between AGAN and ABAO conditions (that is imperfect maintenance is possible) with respect to the maintainable failure modes. We have assumed in Pandey et al. (2013a) that only effective age of the system is affected by imperfect maintenance. We have extended that basic model in this paper with further modifications and a comprehensive modelling and results are presented here. To incorporate the effect of maintenance budget and system age on the imperfect maintenance improvement factors, we have used the cost-age-based imperfect maintenance factors defined in our other work in Pandey et al. (2013b). However, in Pandey et al. (2013b) only one type of hazard rate (that is maintainable hazard rate) is used. In the present paper, we have modified the imperfect maintenance factors, for the age reduction and hazard adjustment, in a way that the two types of hazard rates are accounted for. This new hybrid imperfect maintenance model has been used in this paper and selective maintenance decisions have been made for a series-parallel system.

Selective maintenance is required when it is not possible to perform all feasible maintenance actions due to limited resources. In modern industries and military applications, a system is often required to perform successive missions with a break between them. Because each of the available maintenance options consumes some maintenance resources, like time and cost, an optimal allocation of resources is required. Selective maintenance was introduced by Rice et al. (1998) on a series-parallel system with identical components with limited maintenance time. They assumed a constant hazard rate and that replacement was the only possible maintenance action. Cassady et al. (2001a) considered both the cost and the time as constraints and developed a maintenance optimisation model for series-parallel structures. Cassady et al. (2001b) assumed that a component's lifetime follows the Weibull distribution. Minimal repair and replacement were considered as possible maintenance actions. Although they considered the Weibull distribution, their study was limited in that time was the only resource constraint. Schneider and Cassady (2004) performed selective maintenance for multiple systems simultaneously and termed it as a fleet.

Lust et al. (2009) found that for a system with a large number of components, the optimisation problem became combinatorial in nature and the enumeration method was no longer useful. They found that an evolutionary algorithm was useful in solving 
selective maintenance optimisation problems. Iyoob et al. (2006) solved the resource allocation problem for subsequent missions under selective maintenance. Liu and Huang (2010) assumed that only the effective age of the system is affected by maintenance actions. They did not consider the change in the hazard rate due to maintenance. None of the previous works in selective maintenance considered the presence of the two types of failure modes in the system. In all of the previous works on selective maintenance, it was assumed that all failure modes in the system are maintainable. As explained earlier, some failure modes may not be maintainable. For example gear cracking, bearing failure, fatigue and overall wear of equipment cannot be maintained. However, conveyor belt joint failure, conveyor belt slippages and lubrication loss are maintainable types of failures.

In this paper, we first define the relationship between maintainable and non-maintainable types of failure modes. The maintainable and non-maintainable failure modes for a component are related in a manner that is similar to that described by Castro (2009); however, we have assumed that imperfect maintenance is also possible for a component with respect to maintainable failure modes. We further propose that the hazard rate due to maintainable failure modes is related to the hazard rate due to non-maintainable failure modes up to the current calendar age and not just up to the previous maintenance break as proposed in Castro's (2009) model. We have developed an imperfect maintenance model with the two types of failure modes. We defined a new characteristic constant in this case and have derived from it a system reliability equation. We then formulated the selective maintenance model with the maintainable and nonmaintainable failure modes under hybrid imperfect maintenance. We have aimed to determine the effect of the two types of failure modes on selective maintenance decision making. In this paper, we have considered a multi-component series-parallel system. It has several subsystems connected in a series. A subsystem consists of components connected in a parallel way. The main contribution of this paper can be summarised as:

1 the development of a model that relates maintainable and non-maintainable failure modes considering the current age of the system

2 the development of a hybrid imperfect maintenance model with the two types of failure modes

3 the formulation of a selective maintenance model considering the two types of failures and the determination of its effect on maintenance decisions.

We have formulated the cost and age-based age reduction and hazard adjustment factor for the hybrid imperfect maintenance model when the two types of failure modes are present in the system. To solve this problem, we have assumed that:

1 both the components and the system are in two possible states: working or failed

2 a limited amount of time and budget are available to perform maintenance

3 component states are known by inspection as soon as the components come in for maintenance at the maintenance depot

4 failures of the components are independent of each other in the system

5 the hazard rate of the maintainable failure modes is related to the hazard rate of the non-maintainable failure modes. 
This paper is organised as follows. The introduction is provided in Section 1, followed by PM models in Section 2. System reliability evaluation and selective maintenance modelling is provided in Section 3. The solution methodology is presented in Section 4. Results and discussions are given in Section 5.

\section{Models for PM}

A multi-component series parallel system is studied in this paper. Each component can undergo a range of possible maintenance actions, which are minimal repair, imperfect maintenance /repair or replacement. We assume that there are $s(j=1,2, \ldots, s)$ subsystems and each subsystem $j$ has $n_{j}\left(j^{\prime}=1,2 \ldots, n_{j}\right)$. There are $n$ components in the whole system, that is, $\sum_{j=1}^{s} n_{j}=n$. The state of the component $i$ before maintenance is represented by $Y_{i}$. If a component is working, $Y_{i}=1$, otherwise $Y_{i}=0$. After maintenance, the state of the component $i$ is denoted by $X_{i}$. If a component is working after maintenance then $X_{i}=1$, otherwise $X_{i}=0$. Several discreet levels of maintenance options are available for a component $i$. These options are denoted by $l_{i}, l_{i} \in\left[1,2 \ldots N_{i}\right]$. Here, $l_{i}=1$ is the minimal level of maintenance and $l_{i}=N_{i}$ is the best possible maintenance for a component that is the replacement option. Thus, for the whole system, we have a total of $N=\sum_{i=1}^{n} N_{i} \mathrm{PM}$ actions available. These options for the system are called $l_{\text {system }}\left(l_{\text {system }}=1,2, \ldots, N\right)$. When $Y_{i}=1,1 \leq l_{i} \leq N_{i}-1$ denotes the imperfect maintenance options. When $Y_{i}=0, l_{i}=1$ denotes the minimal repair of the failed component. It represents the component being put into ABAO condition after repair. Other options $2 \leq l_{i} \leq N_{i}-1$ are related to imperfect repair. Depending on the available resources, a different number of components can be selected for maintenance. The selected number of components is denoted by $k(k \leq n)$ and the corresponding set of selected components is denoted as $i_{\text {selected }}=\left[i_{1}, i_{2}, \ldots, i_{k}\right]$. Only one maintenance action can be performed from available $\left[1,2, \ldots, N_{i}\right]$ options for each selected component. We denote these selected maintenance actions as $l_{\text {selected }}=\left[l_{i_{1}}, l_{i_{2}} \ldots, l_{i_{k}}\right]$, obviously $l_{\text {selected }} \subset l_{\text {system }}$. According to the selected maintenance actions during the maintenance break, the hazard rate of the selected components and the total cost and time of maintenance for the system vary.

\subsection{Maintainable and non-maintainable failure modes}

The maintainable and non-maintainable failure modes have associated hazard rates for a component $i$ given by $h_{m, i}$ and $h_{n, i}$, respectively. They are called the maintainable and non-maintainable hazard rates. Failures due to the maintainable failure modes follow the Weibull distribution with the shape and scale parameters $\beta_{m, i}$ and $\alpha_{m, i}$ whereas the non-maintainable failure modes follow the Weibull distribution with the parameters $\beta_{n, i}$ and $\alpha_{n, i}$. Castro (2009) proposed the following relation between the hazard rates due to the maintainable and the non-maintainable failure modes. 


$$
h_{m, i}(t)=h_{0, i}(t-T) \mu^{H_{n, i}(T)},
$$

where $h_{0, i}$ is the maintainable hazard rate when the component $i$ is AGAN, $T$ is the chronological time of the last maintenance, $t$ is the chronological time such that $t>T$, and $H_{n, i}(T)$ is the cumulative hazard rate for the non-maintainable failure modes at time $T$. The maintainable hazard rate in equation (1) is comprised of two parts. The first part $h_{0, i}(t-T)$ represents the effect of the maintainable failure modes while the second part $\mu^{H_{n, i}(t)}$ represents the effect of the non-maintainable failure modes where $\mu$ is a constant.

In the proposed work, we have made two major changes in the Castro (2009) model. The first major change is to consider the effect of the non-maintainable hazard rate up to the current time $t$ rather than considering it up to the last maintenance break $T$. In Castro's (2009) model, the maintenance was instantaneous, which was not realistic. Castro (2009) stated that the hazard rate due to the maintainable failure modes was affected by the non-maintainable hazard rate only up to the time of previous maintenance break. The cumulative hazard rate of the non-maintainable failure modes during the current mission also affects $h_{m, i}(t)$. Therefore, rather than using $H_{n, i}(T)$ as given in equation (1), we use $H_{n, i}(t)$. This change is important because considering the hazard rate only up to the previous maintenance break will give an inaccurate result related to the current time point. This change will ensure that the effect of the cumulative non-maintainable hazard rate up to the current time is incorporated.

The second major change is to consider the effect of imperfect maintenance. Castro (2009) considered that after maintenance, the components are in AGAN condition with respect to the maintainable failure modes. However, it is possible that the components are in between AGAN and ABAO conditions with respect to the maintainable failure modes after maintenance. Hence, a hybrid imperfect maintenance model with the age reduction and hazard adjustment factor is used to replace $h_{0 . i}(t-T)$ in equation (1). This model is explained later in Section 2.3. Another minor change that we incorporated concerns the value of the constant $\mu$. Originally, Castro (2009) defined $\mu>1$; however, this would not cover the case in which the two types of failure modes are not related at all. Therefore, we have extended the definition and have redefined the factor as $\mu \geq 1$. When $\mu=1$, the hazard rates due to the maintainable and non-maintainable failure modes are independent. When $\mu>1$, there exists a relation between these two hazard rates. The higher the value of $\mu$ is, the stronger the relationship is.

The non-maintainable hazard rate of a component is an increasing function of time. However, if a component is replaced during a maintenance break, then the component becomes AGAN with respect to the non-maintainable failure modes as well. If the non-maintainable hazard rate just before maintenance is denoted by $g_{n, i}$, then after maintenance it becomes:

$$
h_{n, i}(t)=\left\{\begin{array}{l}
h_{n, i}^{0}(t-T), \text { if } l_{i}=N_{i} \\
g_{n, i}(t), \quad \text { otherwise }
\end{array}\right.
$$

where $t-T>0$ and $h_{n, i}^{0}$ is the non-maintainable hazard rate for a new component. The cumulative hazard rate for the non-maintainable failure modes up to the current time $t$ after the maintenance can be calculated as: 


$$
H_{n, i}(t)=\left\{\begin{array}{l}
\int_{T}^{t} h_{n, i}(t) d t, \text { if } l_{i}=N_{i} \\
\int_{0}^{t} h_{n, i}(t) d t, \text { otherwise }
\end{array} .\right.
$$

We have explained the hazard rates and the cumulative hazard rate definitions in the imperfect maintenance and the selective maintenance models for the system in later sections.

\subsection{Cost and time of maintenance}

Upon arrival of the system for maintenance, the inspection determines the state $Y_{i}$ and the effective age $B_{i}$ for each component. Depending on the next mission requirement, a component may or may not be selected for maintenance. If selected, the allocated maintenance cost and time depends on the maintenance option $l_{i}$. The maintenance cost of a component $i$ is defined as;

$$
C_{i}\left(l_{i}\right)=c_{i}^{f i x}+c_{i, l_{i}}
$$

where $c_{i}^{f i x}$ is the fixed cost of maintenance and $c_{i, l_{i}}$ is the variable cost of maintenance. The fixed maintenance cost is related to general maintenance actions (dusting, oiling and so on) and setup cost. The variable cost is the cost associated with the selected maintenance option $l_{i}$. For a component, $c_{i, l_{i}}$ for $l_{i}=N_{i}$ equals to the replacement cost denoted by $C_{i}^{R}$. When $Y_{i}=0, c_{i, l_{i}}$ for $l_{i}=1$ denotes the minimal repair cost $C_{i}^{M R}$ and $c_{i, l_{i}}$ for $2 \leq l_{i} \leq N_{i}-1$ denotes the imperfect repair cost. When $Y_{i}=1, c_{i, l_{i}}$ for $1 \leq l_{i} \leq N_{i}-1$ denotes the imperfect maintenance cost. The total maintenance cost for the whole system can be given by:

$$
C=\sum_{i=i_{1}}^{i_{k}} C_{i}\left(l_{i}\right)
$$

Similar to the maintenance cost, the maintenance time for a component can be given as:

$$
T_{i}\left(l_{i}\right)=t_{i}^{f i x}+t_{i, l_{i}},
$$

where $t_{i}^{f i x}$ is the fixed maintenance time and $t_{i, l_{i}}$ is the variable time of maintenance that depends on the selected maintenance option $l_{i}$. For a component, when $l_{i}=N_{i}, t_{i, l_{i}}=T_{i}^{R}$, where $T_{i}^{R}$ is the time required to replace the component, when $Y_{i}=0, t_{i, l_{i}}$ for $l_{i}=1$ denotes the time to perform a minimal repair $T_{i}^{M R}$ and $t_{i, l_{i}}$ for $2 \leq l_{i} \leq N_{i}-1$ denotes the imperfecr repair duration and when $Y_{i}=1, t_{i, l_{i}}$ for $1 \leq l_{i} \leq N_{i}-1$ denotes the time associated with the imperfect maintenance. The total maintenance time for the whole system can be given by: 


$$
T=\sum_{i=i_{1}}^{i_{k}} T_{i}\left(l_{i}\right) .
$$

It should be noted that $i(i=1,2, \ldots, n)$ denotes the components in the system. When $i$ is used as a subscript with a variable/parameter, it shows that the variable/parameter is associated with the component $i$. In this paper, $c_{i, l_{i}}$ represents the variable cost of maintenance related to the maintenance option $l_{i}$ selected for the component $i$. The value $c_{i, l_{i}}$ would be different for different $l_{i}$ values even for the same component $i$. It should also be noted that depending on $l_{i} \in\left[1,2, \ldots, N_{i}\right]$, which may vary from one component to another, the cost, time and hazard rate vary. Similarly, $t_{i, l_{i}}$ shows the variable time of maintenance for the selected component $i$ and maintenance option $l_{i}$ and $h_{m, i}^{\prime}\left(t, l_{i}\right)$ denotes the hazard rate of component $i$ (for the maintainable failure modes) after a maintenance action $l_{i}$ is performed during the maintenance break. The selective maintenance decision is not only about selecting the components for maintenance, but also selecting the maintenance option from all of the available options for that particular component.

Thus, if the maintenance decision for each of the $k$ selected components is known, the total cost and time for maintenance can be calculated. If a component is not selected for maintenance, there is no cost or time involved. Depending on the level of maintenance, the hazard rate of the maintainable failure mode of a component changes. However, there is no effect of maintenance on the hazard rate of the non-maintainable failure mode.

\subsection{Imperfect maintenance/repair model}

Castro (2009) assumed that after a maintenance break, a component is AGAN with respect to maintainable failure modes. However, imperfect maintenance of a component is also possible with respect to the maintainable failure modes. Therefore, it is important to consider the effect of imperfect maintenance in the modelling of maintainable failure modes. We have addressed this issue and a hybrid imperfect maintenance model has been used for this purpose. The hazard rate function $h(t)$ at time $t$ reflects the condition of the component with respect to the maintainable failure modes that depend on its operating history including operating conditions, failure and repairs and PM actions. In a hybrid imperfect PM model,

1 the hazard rate after the PM becomes $a h(x)$ where $a \geq 1$ is the hazard adjustment factor and $x \geq 0$ represents the time elapsed from the PM

2 if the effective age of a component is $t^{\prime}$ immediately before a PM, it reduces to $b t^{\prime}$ immediately after the PM, where $\mathrm{b} \leq 1$ is the age reduction factor.

Given a certain maintainable hazard rate function $h_{m}^{0}(t)$ for $t \in\left(0, T_{1}\right)$, the PM activity during the maintenance interval $\left[T_{1}, T\right]$ changes the hazard rate to $h_{m}^{\prime}(t)$ for $t \geq T$ (Figure 1). The hybrid imperfect PM model can be given as:

$$
h_{m}^{\prime}(T+x)=a h_{m}^{0}\left(b T_{1}+x\right),
$$

where $a \geq 1, b \leq 1$ and $x \geq 0$. 
In this paper, the effective age of a component $i$ before maintenance is given by $B_{i}$. If the hazard adjustment factor and age reduction factor for component $i$ are represented by $a_{i}$ and $b_{i}$, respectively and the maintainable hazard rate before maintenance is $g_{m, i}$, then the first part of the maintainable hazard rate [in equation (1)] after maintenance $h_{0 . i}(t-T)$ is replaced by a hybrid imperfect maintenance-based hazard rate $h_{m, i}^{\prime}\left(t, l_{i}\right)$ as follows:

$$
h_{m, i}^{\prime}\left(t, l_{i}\right)=\left\{\begin{array}{l}
h_{m, i}^{0}(t-T), \text { if } l_{i} \in l_{\text {selected }}, l_{i}=N_{i} \\
a_{i} g_{m, i}\left(b_{i} \cdot B_{i}+(t-T)\right), \text { if } l_{i} \in l_{\text {selected }}, l_{i} \neq N_{i}, \\
g_{m, i}\left(B_{i}+(t-T)\right), \quad \text { otherwise }
\end{array}\right.
$$

where $t-T>0, a_{i} \geq 1$ and $b_{i} \leq 1$. The first part of equation (9) shows that after the replacement the hazard rate of a component is AGAN. The second part of equation (9) shows that after imperfect maintenance/repair of a component, the age reduction and the hazard adjustment factors are used. The third part of equation (9) shows that if a component is not selected for maintenance, its maintainable hazard rate after maintenance remains the same as before maintenance.

Figure 1 Hybrid imperfect maintenance model (see online version for colours)

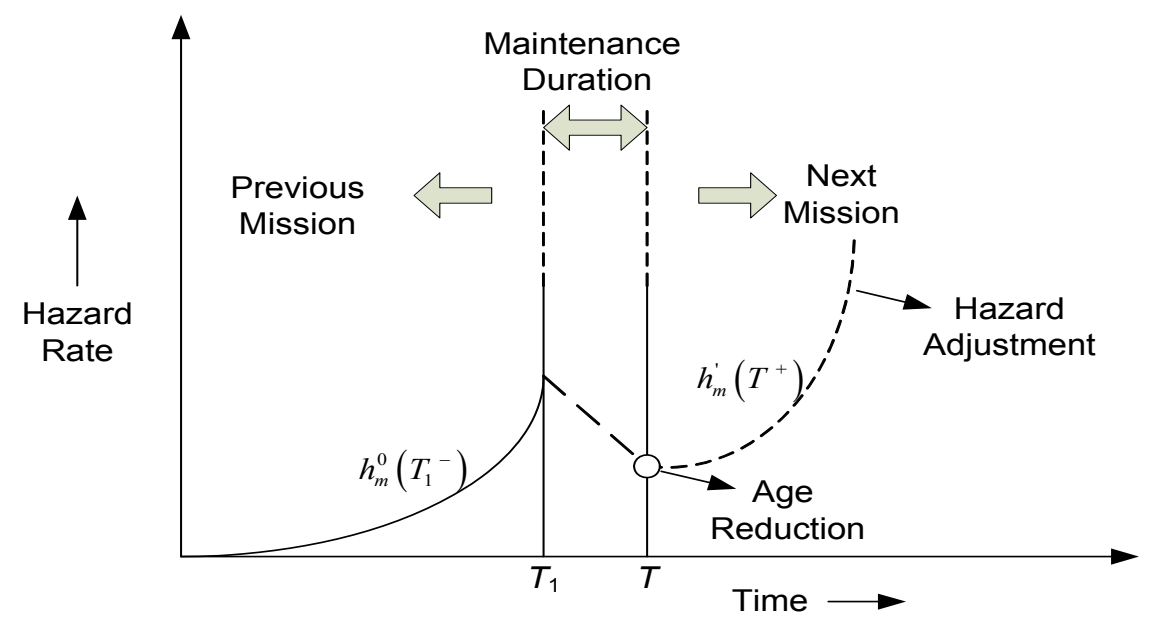

The maintainable hazard rate function in the next mission depends on the hazard rate at the end of the previous mission, the PM activities performed on the component during the maintenance break and the non-maintainable hazard rate function. The hazard adjustment factor $a_{i}$ and the age reduction factor $b_{i}$ depend on the amount of the maintenance budget used (which depends on the maintenance level $l_{i}$ ) as well as on the effective age of the component at the beginning of the maintenance $B_{i}$ (Pandey et al., 2013b). If a component is relatively young, a smaller budget can attain improvement in its condition, but for the same improvement, more budget is expected when the component becomes old. Hence, to incorporate the effect of the amount of budget used and the effective age of the component, a cost-based age reduction factor is used (Pandey et al., 2012, 2013b): 


$$
b_{i}\left(l_{i}, B_{i}\right)=\left\{\begin{array}{l}
1-\left(\frac{C_{i}\left(l_{i}\right)-C_{i}^{M R}}{C_{i}^{R}}\right)^{m\left(B_{i}\right)}, \text { for } Y_{i}=0,1 \leq l_{i}<N_{i} \\
1-\left(\frac{C_{i}\left(l_{i}\right)}{C_{i}^{R}}\right)^{m\left(B_{i}\right)}, \quad \text { otherwise }
\end{array} .\right.
$$

Equation (10) shows that when a component is in the failed state before maintenance and an imperfect repair action is performed on the component, the minimal repair cost is not used in the age reduction because the minimal repair brings the component back to the ABAO condition. In all other cases, the entire maintenance budget is used in improving the condition of the component. Here, $m\left(B_{i}\right)$ is a characteristic constant that denotes the relative age of a component. It is defined as (Pandey et al., 2013b).

$$
m\left(B_{i}\right)=\frac{B_{i}}{\mathrm{MRL}}=\frac{B_{i}}{\left(\frac{\left.\int_{B_{i}}^{\infty} R_{i}(x) d x\right)}{R\left(B_{i}\right)}\right)}=\frac{B_{i} \times R\left(B_{i}\right)}{\int_{B_{i}}^{\infty} R_{i}(x) d x}
$$

Since we have two types of failure modes present in a component, we need to redefine $R_{i}(x)$ as:

$$
R_{i}(x)=\exp \left(-\left(\mu^{\left(x / \alpha_{n, i}\right)^{\beta_{n, i}}}\left(x / \alpha_{m, i}\right)^{\beta_{m, i}}+\left(x / \alpha_{n, i}\right)^{\beta_{n, i}}\right)\right) .
$$

The characteristic constant $m\left(B_{i}\right)$ is defined as the ratio of the effective age $B_{i}$ of the component just before maintenance and its mean, residual life (MRL). If $m\left(B_{i}\right)>1$, it is said to be a relatively old component; otherwise it is relatively young. To check the variation of $m\left(B_{i}\right)$ for a component, we have considered an example with $\mu=1.02$, the Weibull scale parameter and shape parameters $\alpha_{m, i}=300$ and $\beta_{m, i}=1.5$, for the failure distribution of the maintainable failure modes and $\alpha_{n, i}=100, \beta_{n, i}=1.3$ for the non-maintainable failure modes, respectively. When the effective age of the component is 50, 100, 150, 200 and 250 units, respectively, the values of $m\left(B_{i}\right)$ are shown in Figure 2. From Figure 2 it can be seen that as a component becomes older and its effective age $B_{i}$ increases, its $m$ value increases. For $m\left(B_{i}\right)=1$, the effective age of a component is equal to the MRL of the component. In Figure 2, for $B_{i}=50,100$ and 150, $m\left(B_{i}\right)$ is less than 1. Thus at these effective age values, we say that the component is relatively young, whereas for $B_{i}=200$ and 250 when $m\left(B_{i}\right)$ is greater than 1 , the component is relatively old. 
Figure 2 Variation of characteristic constant $m$ with effective age

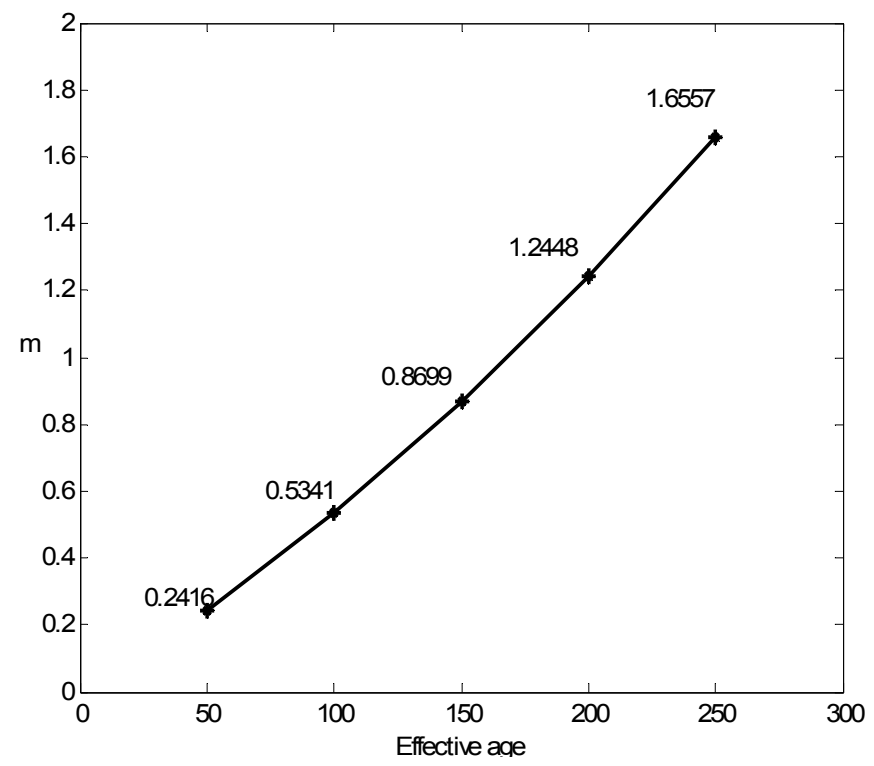

Similar to the age reduction factor, the hazard adjustment factor for the maintainable failure modes is also defined as depending on the maintenance budget used and the effective age of the component (Pandey et al., 2013b):

$$
a\left(l_{i}, B_{i}\right)=\frac{p}{(p-1)+\left(\frac{C_{i}\left(l_{i}\right)}{C_{i}^{R}}\right)^{\frac{1}{m\left(B_{i}\right)}}},
$$

where $p>1$ depends on the maximum hazard adjustment factor that a component can achieve after a maintenance break. The value of this maximum hazard adjustment factor could be estimated through the historical maintenance data about the component (Lie and Chun, 1986) and accordingly, the $p$ value can be decided (Pandey et al., 2013b). The larger the maximum hazard adjustment factor is, the smaller the value of $p$ is.

In equation (9), $h_{m, i}^{\prime}\left(t, l_{i}\right)$ gives the first part of the maintainable hazard rate in equation (1) after the maintenance break, while $\mu^{H_{n, i}(t)}$ is the second part of the maintainable hazard rate in equation (1). When these two parts are put together in equation (1), we obtain the new final expression for the maintainable hazard rate after maintenance:

$$
\begin{aligned}
h_{m, i}\left(t, l_{i}\right)= & h_{m, i}^{\prime}\left(t, l_{i}\right) \mu^{H_{n, i}(t)} \\
= & \left\{\begin{array}{l}
h_{m, i}^{0}(t-T) \mu^{H_{n, i}(t)}, \quad \text { if } l_{i} \in l_{\text {selected }}, l_{i}=N_{i} \\
a_{i} g_{m, i}\left(b_{i} \cdot B_{i}+(t-T)\right) \mu^{H_{n, i}(t)}, \quad \text { if } l_{i} \in l_{\text {selected }}, l_{i} \neq N_{i} \\
g_{m, i}\left(B_{i}+(t-T)\right) \mu^{H_{n, i}(t)}, \quad \text { otherwise }
\end{array}\right.
\end{aligned}
$$

Once a maintenance decision is made and the corresponding cost and time values are determined, we can then determine the achievable system reliability during the next 
mission. Using equations (2) and (14), the cumulative hazard rate for each component can be determined. This cumulative hazard rate can be used to evaluate system reliability as explained below.

\section{Mission reliability evaluation and selective maintenance modelling}

\subsection{Mission reliability evaluation}

The cumulative hazard rate of a component $i$ for the next mission of length $L$ can be defined as:

$$
H_{i, l_{i}}(L)=\int_{T}^{T+L}\left[h_{m, i}\left(t, l_{i}\right)+h_{n, i}(t)\right] d t .
$$

The probability of this component successfully completing the next mission is,

$$
p_{i, l_{i}}=\exp \left(-H_{i, l_{i}}(L)\right)
$$

Thus, the reliability of a component $i$ can be defined as:

$$
R_{i, l_{i}}=p_{i, l_{i}} \times X_{i}
$$

where $X_{i}$ represents the state of the component $i$ after maintenance. Its value is 1 when the component is working; otherwise, it is 0 . Hence, system reliability for the next mission where components within a subsystem are connected in a parallel and the subsystems are connected in a series can be expressed as:

$$
R\left(l_{\text {selected }}\right)=\prod_{j=1}^{s}\left(1-\prod_{j^{\prime}=1}^{n_{j}}\left(1-R_{i, l_{i}}\left(j, j^{\prime}\right)\right)\right)
$$

where $l_{\text {selected }}=\left[l_{i_{1}}, \ldots, l_{i_{k}}\right]$ is an array of $k$ maintenance decisions corresponding to the selected components $\left[l_{1}, \ldots, i_{k}\right]$ during the maintenance break and $R_{i, l_{i}}\left(j, j^{\prime}\right)$ is the reliability of the $i^{\text {th }}$ component in the system, which is also the $j^{\prime \text { th }}$ component in the $j^{\text {th }}$ subsystem. The probability of completing the next mission successfully can be recursively determined for each component using its initial state, calendar age, effective age at the beginning of the mission and the mission duration. Thus, the reliability of the whole system can be determined using equation (18).

\subsection{Selective maintenance modelling}

Our goal has been to determine the components for maintenance and decide the level of maintenance to be performed on these components. We needed to find $i_{\text {selected }}=\left[i_{1}, i_{2} \ldots, i_{k}\right]$ and $l_{\text {selected }}=\left[l_{1}, \ldots, l_{k}\right]$ for the system. We determined the budget $C$ and maintenance time $T$ to be used on the selected components during the maintenance break. These decisions ensure that maximum system reliability is achieved during the next mission. Let the budget constraint on the total maintenance cost during the maintenance break be $C_{0}$ and 
the available maintenance duration be $T_{0}$. The non-linear formulation to maximise the next mission reliability is given as follows:

Objective:

$$
\operatorname{Max} R\left(l_{\text {selected }}\right)=\prod_{j=1}^{s}\left(1-\prod_{j^{\prime}=1}^{n_{j}}\left(1-R_{i, l_{i}}\left(j, j^{\prime}\right)\right)\right)
$$

Subject to:

$$
\begin{aligned}
& \sum_{i=i_{1}}^{i_{k}} C_{i}\left(l_{i}\right) \leq C_{0} \\
& \sum_{i=i_{1}}^{i_{k}} T_{i}\left(l_{i}\right) \leq T_{0} \\
& V_{i}=\left\{\begin{array}{l}
1, \text { if } l_{i} \in l_{\text {selected }} \\
0, \text { otherwise }
\end{array}\right. \\
& X_{i}=\left\{\begin{array}{l}
V_{i}, \text { if } Y_{i}=0 \\
1, \text { otherwise }
\end{array}\right. \\
& 1 \leq l_{i} \leq N_{i}
\end{aligned}
$$

In this formulation, constraints (20) and (21) set the limitations of the available budget and time available to perform maintenance and constraints (22) and (23) set the components' states at the beginning of the next mission depending on their states at the end of the previous mission and the maintenance actions performed. If a component is selected for maintenance, in other words, if $l_{i} \in l_{\text {selected }}, V_{i}$ is 1 ; otherwise it is zero. Now, from equation (23), if $Y_{i}=0, X_{i}$ is equal to $V_{i}$, i.e., $X_{i}=1$ if $l_{i} \in l_{\text {selected }}$; otherwise $X_{i}=0$. In all other cases, when a component was working before maintenance, it will remain in a working state after a maintenance break whether or not it is selected for maintenance. State $X_{i}$ affects the system reliability evaluation as given in equation (17). Constraint (24) shows the possible maintenance levels for a component $i$.

\section{Solution methodology}

To solve the nonlinear optimisation problem of selective maintenance, we have used an evolutionary algorithm. Evolutionary algorithms for example, genetic algorithm, differential evolution (DE) and so forth are easy to use and adaptable to the problem (Levitin and Lisnianski 2000; Lust et al. 2009; Liu and Huang, 2010; and others). DE (Brest et al., 2006) is used here to solve the problem. DE starts with a population and this population evolves to find the optimal/near optimal solution. A population is a set of solution strings. A solution string is represented by $S$ where $S=\left(s_{1}, s_{2}, \ldots, s_{K_{\max }}\right)$. Here $K_{\max }$ is the number of elements in a solution string. The value $K_{\max }$ is chosen to be greater than or equal to the total possible number of maintenance actions for the whole system $N$. Each element of the string $\left(s_{1}, s_{2}, \ldots, s_{K_{\max }}\right)$ is one of the $N$ PM actions available for the 
system. In each element's position, maintenance action is randomly generated from the available $N$ options from the whole system. Thus it is possible that in a solution string, PM actions related to one component appear more than once. We will therefore consider only the first appearing PM action for a component.

The number of PM actions for the system that satisfies the constraints given in equations (20)-(24) may vary from one solution to another depending on the set of maintenance actions $\left(l_{i}\right)$ in that solution string. This is because different maintenance actions consume different amounts of resources and they affect system reliability differently. We need to assign a number to represent this useful number of PM actions in a solution string. We denoted this position in the solution string by $P$. Only useful parts of the solution string $\left[s_{1}, s_{2}, \ldots s_{P}\right]$ will define the PM plan, while part of solution string $\left[s_{P+1}, s_{P+2}, \ldots, s_{K_{\max }}\right]$ will not contribute to the final PM solution. The elements of the string from $s_{p+1}$ to $s_{K_{\max }}$ do not affect the objective function or the constraints, but they may affect the offspring by participating in the DE steps.

The following procedure is used to determine the objective function and constraint values of an arbitrary integer solution string $S=\left[s_{1}, s_{2}, \ldots, s_{K_{\max }}\right]$.

1 Define $K_{\max }$. Set total $\operatorname{cost} C=0$, total time $T=0$, system reliability $R=1$.

2 Define sets $i_{\text {selected }}=\phi$ and $l_{\text {selected }}=\phi$.

3 For $K=1$, the first element $s_{1}$ corresponds to the maintenance action $l_{i}$ for a component $I$. Thus component $i$ is the first component to be selected; hence for $k=1$, $i_{k=1}=i$ and $i_{\text {selected }}=\bigcup i_{k}, l_{\text {selected }}=\bigcup l_{i_{k}}$. Here $k$ denotes the order of the selected components for maintenance. Corresponding to the decision $l_{\text {selected }}$, update the total cost $C$ using equation (5), total maintenance time $T$ using equation (7) and system reliability $R$ using equation (18).

4 If $C<C_{0}, T<T_{0}, K<K_{\max }$ and $k<n$, set $K=K+1$, go to step 6 .

5 If $C \geq C_{0}$ or $T \geq T_{0}$ or $K>K_{\max }$ or number of elements in $i_{\text {selected }}=n$ (total number of components), go to step 9.

6 For $K, s_{K}$ represents a maintenance action $l_{i}$ for component $i$, check if $i \in i_{\text {selected }}$, i.e., check whether a component is already selected for maintenance in the solution string.

7 If step 6 is true, then $K=K+1$; go back to step 6. Otherwise go to step 8 .

8 For $K$ and maintenance action $l_{i}$ given by element $s_{K}, k=k+1, i_{k}=i, i_{\text {selected }}=\bigcup i_{k}$ and $l_{\text {selected }}=\bigcup l_{i_{k}}$. Update the total $\operatorname{cost} C$, total maintenance time $T$ and the system reliability $R$. Go to step 4 .

9 Stop. Reliability $R$ is the final value for the given solution string. Finally, $l_{\text {selected }}=\bigcup l_{i_{k}}$ gives the maintenance option selected for the system and $i_{\text {selected }}=\bigcup i_{k}$ gives the components selected corresponding to $l_{\text {selected }}$. If $K<K_{\max }$, then $P=K$. 
For example, two components are connected in a series and for each component, three maintenance options are available; hence, $N_{i}=3$ for both components and $N=\sum_{i=1}^{2} N_{i}=6$. In this example, we define $K_{\max }=N=6$. If a randomly generated solution string is given as [ $\left[\begin{array}{lllll}2 & 3 & 4 & 6 & 5\end{array}\right.$ 4], then for $K=1, s_{1}$ is $l_{i}=2$ and the component is $i=1$. For this selection we have, $k=1$ and the first selected component is $i_{1}=1$. Thus, $i_{\text {selected }}=\left[i_{1}\right]=[1]$ and $l_{\text {selected }}=[2]$. We assume that the cost and time calculated for this decision is within constraint limits. Now for $K=2, l_{i}=3$. However, corresponding to this maintenance decision the component $i=1$ is already selected; hence, we do not consider $s_{2}$ in the PM solution. We move to the next $K=K+1=2+1=3$. For $s_{3}, l_{i}=4$ related to the component $i=2$. Because $i=2 \notin i_{\text {selected }}$, we update $k=1+1=2$ and the second selected component becomes $i_{2}=2$. Thus $i_{\text {selected }}=\left[i_{1}, i_{2}\right]=[1,2]$ and $l_{\text {selected }}=[2,4]$. We calculate the cost, time and the system reliability corresponding to this maintenance decision $l_{\text {selected }}$. Equations (5), (7) and (18) are used to calculate the maintenance cost, time and system reliability for any $l_{\text {selected. }}$. No other solution can be selected, as maintenance decisions corresponding to both components in the system are already realised.

\section{Results and discussion}

To demonstrate the applicability of the above model, we have used an example of a coal transportation system in a power generation system that was also employed by Liu and Huang (2010). This coal transportation system is used to supply coal to a boiler in a power station. It includes five basic subsystems, as shown in Figure 3.

Figure 3 Block diagram of a coal transportation system

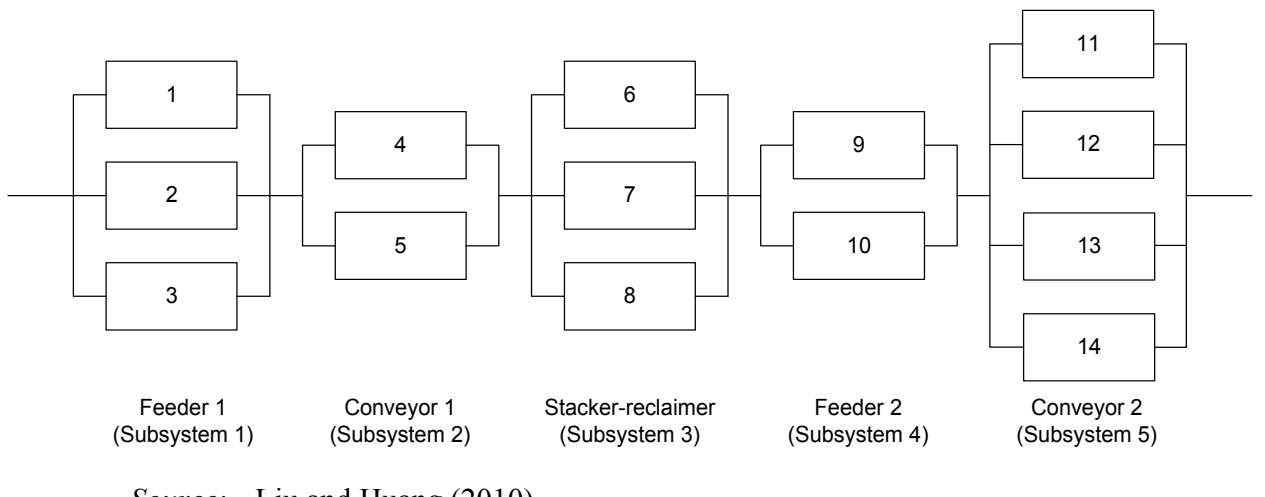

Source: Liu and Huang (2010)

Feeder 1 transfers coal from the bin to conveyor 1. Conveyor 1 transports the coal from feeder 1 to the stacker reclaimer, which lifts the coal up to the burner level. Feeder 2 then loads conveyer 2, which transfers the coal to the boiler's burner feeding system. Each of the subsystems consists of a different number of components and each component has different parameters, as shown in Table 1. 
Table 1 System parameters

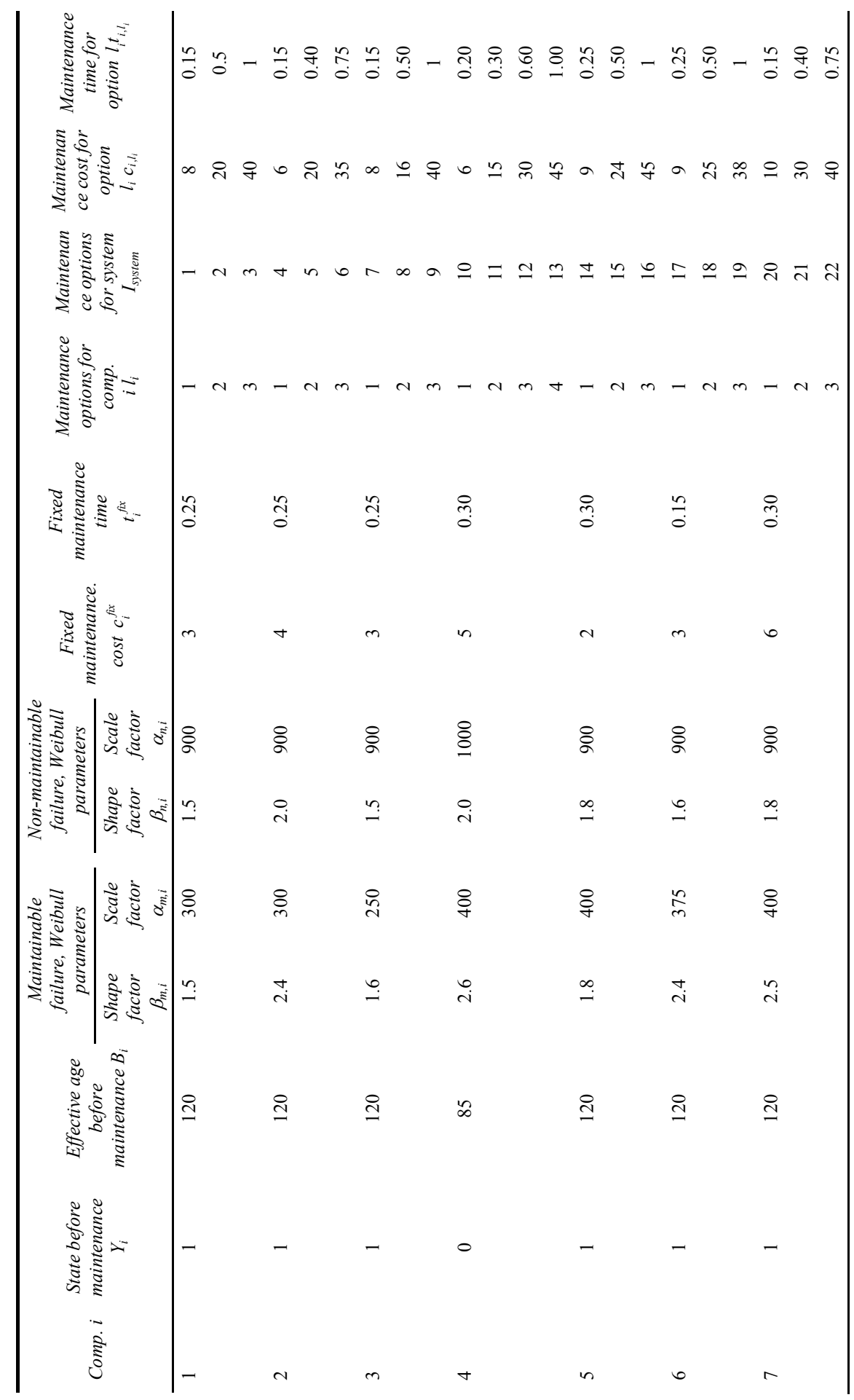


Table 1 System parameters (continued)

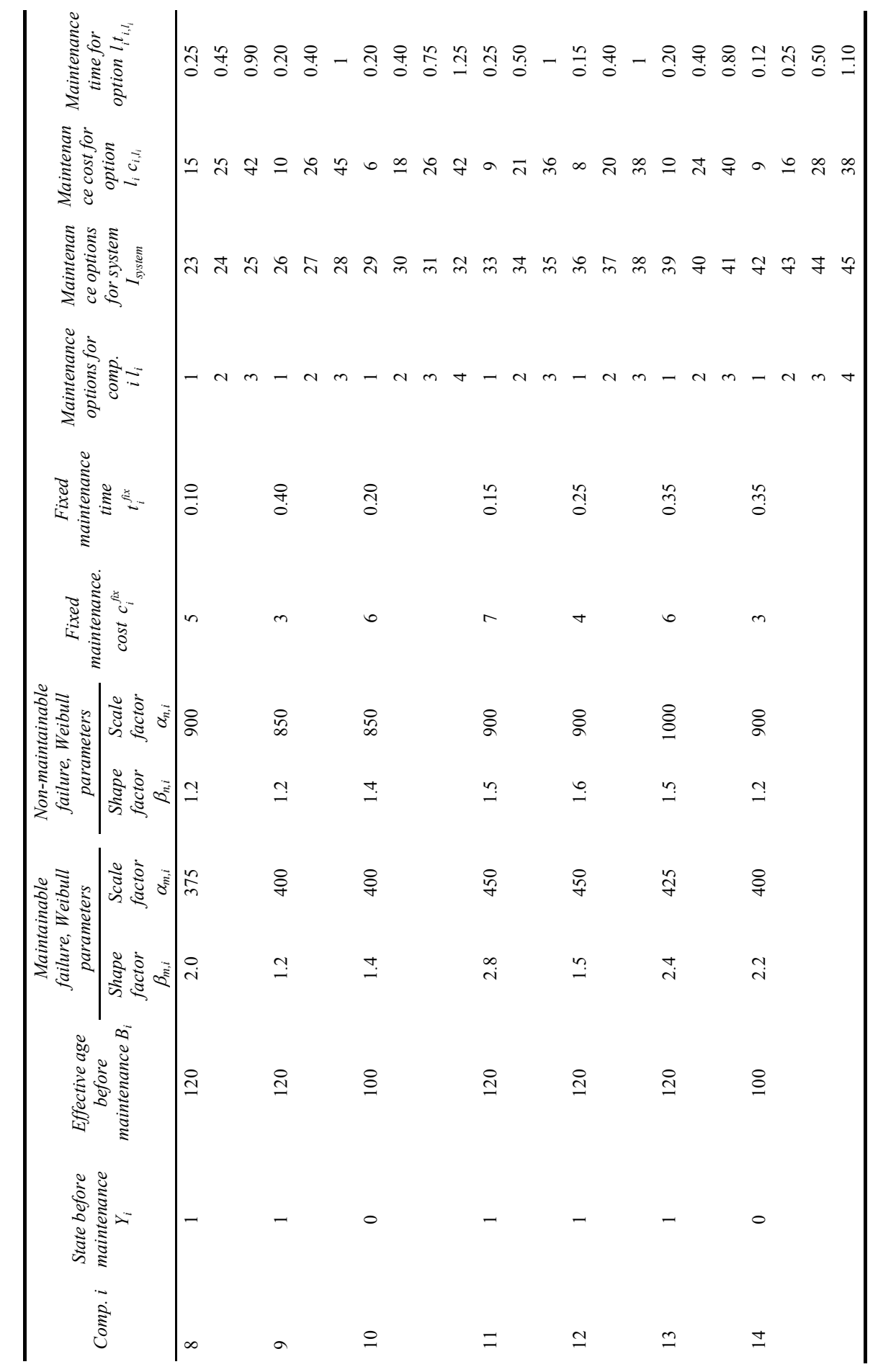


There are two possible imperfect repair/maintenance levels for each component. For a working component (i.e., $\left.Y_{i}=1\right) l_{i}=[1,2,3]$. Here, $l_{i}=1,2$ denote two imperfect maintenance levels and $l_{i}=3$ denotes replacement of the component. For a failed component $\left(Y_{i}=0\right)$, minimal repair is also an option. In this case, $l_{i}=[1,2,3,4]$ are the possible maintenance levels. Here, $l_{i}=1$ represents the minimal repair, $l_{i}=2,3$ are the imperfect repair levels and $l_{i}=4$ is the replacement of the component.

We consider that the system has stopped for its first maintenance break after 120 days of the mission. The next mission duration is 90 days. In this example, one cost unit is $\$ 1,000$ and one time unit is 1 day. We assume that $p=20$ in equation (13) and is similar to Castro (2009) $\mu=1.02$ in equation (14) for all components. The effect of the imperfect maintenance /repair on selective maintenance decision making, resource constraints and their sensitivity, the maintainable and non-maintainable failure modes and their relation are analysed in detail. Point wise discussions are provided below. In the following discussion, IM is imperfect maintenance, IR is imperfect repair, MR is minimal repair and $\mathrm{CR}$ is component replacement.

\subsection{Effect of resource constraints}

We first determine the effect of the resource constraints on selective maintenance decision making and analyse resource sensitivity. We first consider $C_{0}=400$ units as the only resource constraint. The results are shown in Table 2 . It can be seen from Table 2 that when cost is the only constraint, ten components are selected during the maintenance break. Components 2, 3, 4, 5, 6, 7, 9 and 10 undergo replacement while imperfect maintenance is performed on component 11. Imperfect repair is performed on failed component 4 . No maintenance is performed on the other four components, components 1 , 8,12 and 13. In this case, out of the available 400 cost units, 397 units are utilised and 10.90 time units are used in maintenance. When we introduce time as an additional constraint and keep $T_{0}=7$ units, the maintenance decision is changed. The results are given in Table 3.

Table 2 Only cost as constraint

\begin{tabular}{lc}
\hline Maintenance decision, $l_{\text {selected }}$ & $6,9,13,16,19,22,28,32,33,43$ \\
Maintenance action & $\mathrm{CR}, \mathrm{CR}, \mathrm{CR}, \mathrm{CR}, \mathrm{CR}, \mathrm{CR}, \mathrm{IM}, \mathrm{IR}$ \\
Components selected, $i_{\text {selected }}$ & $2,3,4,5,6,7,9,10,11,14$ \\
Total time consumed, $T$ & 10.90 units \\
Total cost consumed, $C$ & 397 \\
System reliability, $R$ & $96.04 \%$ \\
\hline
\end{tabular}

Note: $C_{0}=400$ units

With time as an additional constraint, only 250 units out of a total budget of 400 units and 6.80 units of time are used. The remaining 150 cost units are unused because there is no time available to perform maintenance/repair of any component that can further improve system reliability. When both constraints are considered, only six components are selected due to the time limitation and no maintenance/repair action is performed on the remaining eight components. It is evident that with an increase in the amount of the available resources, the maintenance decision changes. This also demonstrates that maintenance decisions are sensitive to resource variation. It is thus important for a 
maintenance manager to perform a sensitivity analysis before making a final maintenance decision.

Table 3 Both cost and time as constraint

\begin{tabular}{lc}
\hline Maintenance decision, $l_{\text {selected }}$ & $6,13,22,28,32,43$ \\
Maintenance action & $\mathrm{CR}, \mathrm{CR}, \mathrm{CR}, \mathrm{CR}, \mathrm{CR}, \mathrm{IR}$ \\
Components selected, $i_{\text {selected }}$ & $2,4,7,9,10,14$ \\
Total time consumed, $T$ & 6.80 units \\
Total cost consumed, $C$ & 250 units \\
System reliability, $R$ & $95.09 \%$ \\
\hline
\end{tabular}

Notes: $C_{0}=400$ units, $T_{0}=7$ units

To perform a sensitivity analysis, we have varied $C_{0}$ as $50,100,200,300$ and 400 units whereas $T_{0}$ as 3,5, 7 and 9 units. The variation is shown in Figure 4. When $C_{0}=50$ units, the maximum achievable system reliability is 0.8772 for all values of $T_{0}$. For this budget, the selected maintenance actions are imperfect maintenance of components 6 and 8 , which need 42 cost units. Although only 0.95 time unit is required for this maintenance, no further maintenance is possible because no budget is available for any other maintenance that can improve system reliability. Similarly, for $C_{0}=100$ units, the maximum achievable system reliability is 0.9113 . This reliability can be achieved when $T_{0}=3$. Any further increase in time is not useful. Thus for each of the given budgets, there is a limit of time beyond which no further increase in system reliability is possible.

Figure 4 Sensitivity analysis of available budget and maintenance time (see online version for colours)

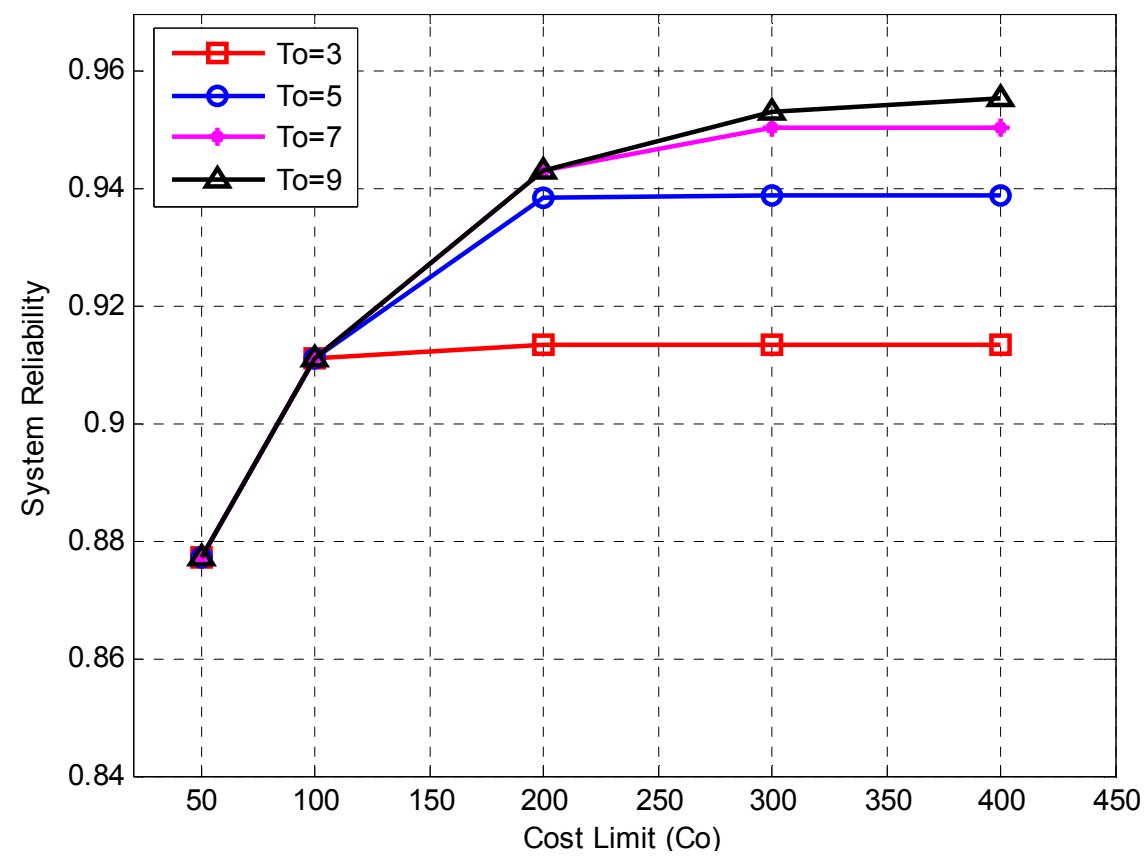


Similar observations can be found for the time limit as well. For $T_{0}=3$ units, system reliability is 0.8772 for $C_{0}=50$ units, 0.9113 for $C_{0}=100$ units and 0.9134 for $C_{0}=200$ units. However, any further increase in $C_{0}$ will not increase system reliability because there is no time available to perform other maintenance options. This demonstrates that a maintenance manager needs to be clear about the relative consumption of resources for each of the possible maintenance actions so that an optimal allocation of resources can be performed. It can also be seen that for $T_{0}=9$ units, an increase in the maintenance budget from 300 to 400 units leads to an increase in system reliability of less than $1 \%$. Hence, the maintenance manager can decide whether it is beneficial to assign extra resources for the minimal increase in system reliability.

\subsection{Comparing replacement/minimal repair and imperfect maintenance /repair as maintenance options}

When only replacement and minimal repair are considered as possible maintenance actions, only 17 options are available for the systems, which are 14 replacement options and three minimal repair options for the failed components 4, 10 and 14. Let us assume that 500 units of the budget are available for maintenance and only 13 days are available to perform maintenance. The maintenance crew has to determine the components and the maintenance actions on these selected components so that maximum reliability is achieved during the next mission. The results are shown in Table 4.

Table 4 Only replacement and minimal repair are possible

\begin{tabular}{lc}
\hline Maintenance decision, $l_{\text {selected }}$ & $3,6,9,13,16,19,22,25,28,32,42$ \\
Maintenance option & $\mathrm{CR}, \mathrm{CR}, \mathrm{CR}, \mathrm{CR}, \mathrm{CR}, \mathrm{CR}, \mathrm{CR}, \mathrm{CR}, \mathrm{CR}, \mathrm{CR}, \mathrm{MR}$ \\
Components selected, $i_{\text {selected }}$ & $1,2,3,4,5,6,7,8,9,10,14$ \\
Total time consumed, $T$ & 12.62 units \\
Total cost consumed, $C$ & 311 units \\
System reliability, $R$ & $92.68 \%$ \\
\hline
\end{tabular}

Notes: $C_{0}=500$ units, $T_{0}=13$ units

Table 4 depicts that 11 components (component \#s 1, 2, 3, 4, 5, 6, 7, 8, 9, 10 and 14) are selected for maintenance. Minimal repair is performed on component 14 while all other selected components are replaced. The maximum achievable system reliability is $92.68 \%$. It is interesting to note that out of 500 units of budget, only 311 units are used. The remaining 189 units of budget are unused because there is no time available to perform additional maintenance. This verifies that it is critical for a maintenance manager to determine the effect of maintenance resources on the final maintenance decision and accordingly decide on resource requirements. More time is required to better utilise the remaining budget. No maintenance is done on components 11,12 and 13 .

When imperfect maintenance /repair is introduced in addition to replacement and minimal repair, higher system reliability is achieved (Table 5). Table 5 shows that when imperfect maintenance is introduced in addition to replacement, system reliability is $96.26 \%$, an increase of about $4 \%$ t. This illustrates that the use of imperfect maintenance /repair is beneficial compared to replacement only. Higher system reliability is possible because the introduction of imperfect maintenance /repair enables using most of the available resources, which may not be possible if only replacement is performed. Another 
reason for the improvement in reliability due to imperfect maintenance is that it allows an option where more components can be imperfectly maintained.

Table 5 Imperfect maintenance /repair, replacement and minimal repair are possible

\begin{tabular}{lc}
\hline Maintenance decision, $l_{\text {selected }}$ & $3,6,9,13,16,19,22,23,28,32,34,44$ \\
Maintenance option & $\mathrm{CR}, \mathrm{CR}, \mathrm{CR}, \mathrm{CR}, \mathrm{CR}, \mathrm{CR}, \mathrm{CR}, \mathrm{CR}, \mathrm{MR}, \mathrm{CR}, \mathrm{CR}, \mathrm{IR}$ \\
Components selected, $i_{\text {selected }}$ & $1,2,3,4,5,6,7,8,9,10,11,14$ \\
Total time consumed, $T$ & 13.00 units \\
Total cost consumed, $C$ & 484 units \\
System reliability, $R$ & $96.26 \%$ \\
\hline
\end{tabular}

Notes: $C_{0}=500$ units, $T_{0}=13$ units

\subsection{Effect of maintainable and non-maintainable failure modes relation}

In this paper, the hazard rate due to the maintainable failure modes is related to the hazard rate due to the non-maintainable failure modes. We have also investigated how the relationship between these two failure modes affects the final maintenance decision. We can rework the example with $C_{0}=400$ units and $T_{0}=7$ units; however, this time we assume that there is no relation between maintainable and non-maintainable failure modes, i.e., $\mu=1$. The results are shown in Table 6 . Tables 3 and 6 suggest that the maintenance decisions remain the same for both cases. To check the sensitivity of the selective maintenance decision with respect to the factor $\mu$ value, we changed the value of $\mu$ to $1,5,10,15$ and 20 , where $C_{0}=400$ units and $T_{0}=7$ units. A larger value of $\mu$ denotes a stronger relationship between the two types of hazard rates. The results are shown in Figure 5.

Table 6 No relation between the failure modes

\begin{tabular}{lc}
\hline Maintenance Decision, $l_{\text {selected }}$ & $6,13,22,28,32,43$ \\
Maintenance action & $\mathrm{CR}, \mathrm{CR}, \mathrm{CR}, \mathrm{CR}, \mathrm{CR}, \mathrm{IR}$ \\
Components selected, $i_{\text {selected }}$ & $2,4,7,9,10,14$ \\
Total time consumed, $T$ & 6.80 units \\
Total cost consumed, $C$ & 250 units \\
System reliability, $R$ & $95.10 \%$ \\
\hline
\end{tabular}

Notes: $C_{0}=400$ units, $T_{0}=7$ units

Figure 5 depicts that for the given example, system reliability is not very sensitive to the factor $\mu$ value in equation (14). There is a change of only about $2 \%$ in system reliability when $\mu$ changes from 1 to 20 because the values of the cumulative hazard rate due to non-maintainable failure modes is small (much less than 1); therefore, an even larger increase in the factor does not result in too much variation in system reliability. However, if we change the value of $\alpha_{n, i}$ for all components to 500 in Table 1, for the factor value of 5 , system reliability reduces to 0.8843 . This indicates that when a component is old or its non-maintainable hazard rate is high, the value of the factor $\mu$ becomes important. It is therefore important to evaluate the value of the factor carefully for the system. Another interesting finding is that for all of the above cases, the maintenance decision remained the same: the replacement of components $2,4,7,9,10$ and IR of component 14 . This was 
because, for the given cost and time constraints, maximum system reliability was achieved for the above decision for each of the $\mu$ values.

Figure 5 System reliability versus the factor $\mu$

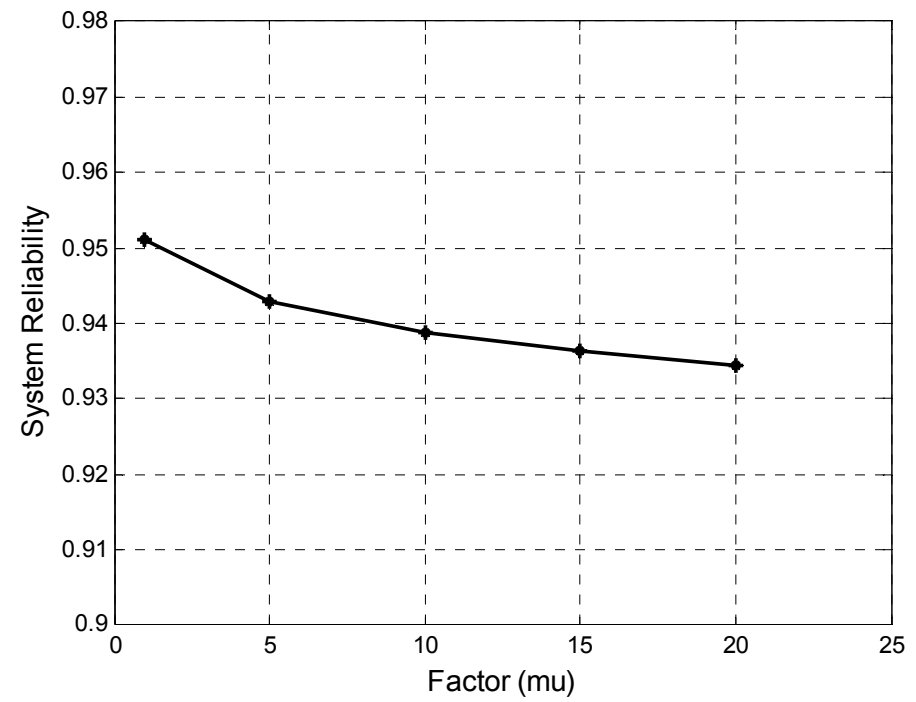

We have improved the Castro (2009) model by changing the value of $H_{n, i}(T)$ to $H_{n, i}(t)$ in calculating the maintainable hazard rate as given in equation (14). Because $T$ is the time of the end of the last maintenance break, Castro's (2009) model did not consider the current condition of the component in determining the maintainable hazard rate. We have considered the instantaneous time point $t$ in order to find the effect of the non-maintainable hazard rate on the maintainable hazard rate. Consideration of instantaneous time $t$ in the non-maintainable hazard rate for calculating the instantaneous maintainable hazard rate during the next mission will give more accurate results compared to the case when a fixed value (the time of the last maintenance $T$ ) is used. This will also affect system reliability. When we use $H_{n, i}(T)$ for $C_{0}=400$ units and $T_{0}=7$ units with the mission duration of 90 time units and the factor $\mu$ value of 5 , the best possible maintenance decision, the replacement of the components $2,4,7,9$ and 10 and IR of component 14 , generates a system reliability of 0.9453 . However, this system reliability value is reduced to 0.9429 when $H_{n, i}(t)$ is used. Since the cumulative hazard rate up to the previous maintenance break is smaller, it produces a higher estimate of system reliability than the actual reliability in the current time. Hence, it may affect maintenance decisions where reliability is important and the decision is based on system reliability evaluation.

It can be seen from the above discussion that considering the imperfect maintenance /repair is advantageous over only minimal repair/replacement as maintenance options. The former allows the maximum utilisation of available resources. Also, selective maintenance decisions depend on the number of resource constraints. We have found it to be advantageous to perform a sensitivity analysis so that resource allocation can be done wisely in order to attain desired reliability. The dependency between the maintainable and non-maintainable failure modes also affects maintenance decisions. Hence, it is useful to find an appropriate value of the factor $\mu$. We have also found that considering the effect of the non-maintainable hazard rate only up to the previous maintenance break 
may be misleading and will give an optimistic reliability value that is higher than the actual system reliability. This may lead to failure of the system while the maintenance crew believes that the system is more reliable than it actually is.

\section{Conclusions}

In this paper, a selective maintenance policy has been established for a multi-component, series-parallel system experiencing the two types of failure modes: maintainable and non-maintainable. There are hazard rates corresponding to the both types of failure modes. We have proposed a formulation to relate these hazard rates when imperfect maintenance /repair of the components is possible. A hybrid imperfect maintenance model has been used to reflect the effect of age reduction and hazard adjustment. Costand age-based imperfect maintenance /repair factors have been used. The changes are incorporated into the imperfect maintenance model in order to consider both types of the hazard rates and their relationship.

A comparison has been provided when only cost or both cost and time are used as the available resources. A resource sensitivity analysis has also been performed. We have found that it is useful to include imperfect maintenance /repair as maintenance options along with replacement/minimal repairs. This results in better utilisation of resources and yields higher system reliability. The relationship between the maintainable and non-maintainable failure modes is found to be important, which may affect final maintenance decisions. The stronger the relationship is the smaller achievable system reliability is for the next mission. The effect of the failure modes on successive missions and maintenance scheduling can be evaluated in the future. It is also important to consider the effect of the non-maintainable failure modes up to the current time rather than only up to the previous maintenance break. Otherwise, it may mislead maintenance decisions and indicate reliability that is higher than actual system reliability. In the future, a trade-off between the multiple resources and system reliability can be studied using a multi-objective formulation.

\section{Acknowledgements}

The authors are thankful to the anonymous reviewers and editors for their comments, which helped in improving the quality of the paper. This research was supported by the Natural Sciences and Engineering Research Council of Canada (NSERC).

\section{References}

Brest, J., Greiner, S., Boskovie, B., Mernik, M. and Zumer, V. (2006) 'Self-adapting control parameters in differential evolution: a comparative study on numerical benchmark problems', IEEE Transactions on Evolutionary Computation, Vol. 10, No. 6, pp.646-657.

Cassady, C.R., Pohl, E.A. and Murdock Jr., W.P. (2001a) 'Selective maintenance modeling for industrial systems', Journal of Quality in Maintenance Engineering, Vol. 7, No. 2, pp.104-117. 
Cassady, C.R., Murdock Jr., W.P. and Pohl, E.A. (2001b) 'Selective maintenance for support equipment involving multiple maintenance actions', European Journal of Operational Research, Vol. 129, No. 2, pp.252-258.

Castro, I.T. (2009) 'A model of imperfect preventive maintenance with dependent failure modes', European Journal of Operational Research, Vol. 196, No. 1, pp.217-224.

Chen, M., Xu, C. and Zhou, D. (2012) 'Maintaining systems with dependent failure modes and resource constraints', IEEE transactions on Reliability, Vol. 61, No. 2, pp.440-451.

Høyland, A. and Rausand, M. (1994) System Reliability Theory: Models, Statistical Methods and Applications, Wiley, London.

Huynh, K.T., Barros, A., Bérenguer, C. and Castro, I.T. (2011) 'A periodic inspection and replacement policy for systems subject to competing failure modes due to degradation and traumatic events', Reliability Engineering and System Safety, Vol. 96, No. 4, pp.497-508.

Iyoob, I.M., Cassady, C.R. and Pohl, E.A. (2006) 'Establishing maintenance resource levels using selective maintenance', Engineering Economist, Vol. 51, No. 2, pp.99-114.

Kim, D.S., Ok, S.Y., Song, J. and Koh, H.M. (2013) 'System reliability analysis using dominant failure modes identified by selective searching technique', Reliability Engineering and System Safety, Vol. 119, No. 1, pp.316-331.

Levitin, G. and Lisnianski, A. (2000) 'Optimization of imperfect preventive maintenance for multi-state systems', Reliability Engineering and Systems Safety, Vol. 67, No. 2, pp.193-203.

Lie, C.H. and Chun, Y.H. (1986) 'An algorithm for preventive maintenance policy', IEEE Transactions of Reliability, Vol. 35, No. 1, pp.71-75.

Lin, D., Zuo, M.J. and Yam, R.C.M. (2000) 'General sequential imperfect preventive maintenance models', International Journal of Reliability, Quality and Safety Engineering, Vol 7, No. 3, pp.253-266.

Lin, D., Zuo, M.J. and Yam, R.C.M. (2001) 'Sequential imperfect preventive maintenance models with two categories of failure modes', Naval Research Logistics, Vol. 48, No. 2, pp.172-183.

Liu, T.B., Kang, J.S., Li, Y.Y. and Luo, G.K. (2012) 'Imperfect preventive maintenance model with two modes of failure', International Conference on Information Management, Innovation Management and Industrial Engineering, pp.492-495.

Liu, X. (2012) 'Planning of accelerated life tests with dependent failure modes based on a gamma frailty model', Technometrics, Vol. 54, No. 4, pp.398-409.

Liu, Y. and Huang, H.Z. (2010) 'Optimal selective maintenance strategy for MSS under imperfect maintenance', IEEE Transactions on Reliability, Vol. 59, No. 2, pp.356-367.

Lust, T., Roux, O. and Riane, F. (2009) 'Exact and heuristic methods for the selective maintenance problem', European Journal of Operational Research, Vol. 197, No. 3, pp.1166-1177.

Malik, M.A.K. (1979) 'Reliable preventive maintenance scheduling', AIIE Transactions, Vol. 11, No. 3, pp.221-228.

Nakagawa, T. (1988) 'Sequential imperfect preventive maintenance policies', IEEE Transactions of Reliability, Vol. 37, No. 3, pp.295-298.

Nguyen, D.G. and Murthy, D.N.P. (1981) 'Optimal preventive maintenance policies for repairable systems’, Operations Research, Vol. 29, No. 6, pp.1181-1194.

Pandey, M., Zuo, M.J. and Moghaddass, R. (2012) 'Selective maintenance for binary systems using age-based imperfect repair model', 2012 International Conference on QR2MSE, pp.385-389.

Pandey, M., Zuo, M.J. and Cuong, D.D. (2013a) 'Selective maintenance for a multi-component system with two types of failure modes under age-based imperfect maintenance', 19th ISSAT International Conference: Reliability and Quality in Design, August 5-7, Honolulu, Hawaii, USA.

Pandey, M., Zuo, M.J., Moghaddass, R. and Tiwari, M.K. (2013b) 'Selective maintenance for binary systems under imperfect repair', Reliability Engineering and System Safety, Vol. 113, No. 1 , pp.42-51. 
Pham, H. and Wang, H. (2003) 'Optimal imperfect maintenance models', in Pham, H. (Ed.): Handbook of Reliability Engineering, pp.397-414, Springer, London.

Rice, W.F., Cassady, C.R. and Nachlas, J.A. (1998) 'Optimal maintenance plans under limited maintenance time', Proceedings of the Seventh Industrial Engineering Research Conference, Banff, Canada.

Schneider, K. and Cassady, C.R. (2004) 'Fleet performance under selective maintenance', The Annual Reliability and Maintainability Symposium, St. Louis, USA.

Wang, H.Z. (2002) 'A survey of maintenance policies of deteriorating systems', European Journal of Operational Research, Vol. 39, No. 3, pp.469-489.

Yang, Q., Hong, Y., Chen, Y. and Shi, J. (2012) 'Failure profile analysis of complex repairable systems with multiple failure modes', IEEE Transactions on Reliability, Vol. 61, No. 1, pp.180-191.

Zequeira, R.I. and Bérenguer, C. (2006) 'Periodic imperfect preventive maintenance with two categories of competing failure modes', Reliability Engineering and System Safety, Vol. 91, No. 4, pp.460-468. 